\title{
Recent changes to the Author Notes for Powder Diffraction
}

With the increase of submissions of powder diffraction data, the editors of Powder Diffraction journal have recently added a new manuscript type called Data Reports, and made changes to the following sections of the Author Notes for the submission of New Diffraction Data and Rapid Communications. Please see the changes below.

The Author Notes are located in full online at: http://www. icdd.com/resources/pdj/authors.htm

\section{CATEGORIES OF MANUSCRIPTS}

(3) New Diffraction Data are short articles which present new or significantly improved powder diffraction patterns and/or crystal structure determination on crystalline materials of interest to science and industry. A New Diffraction Data paper should include chemical name, chemical formula sample source and purity, experimental documentation, analysis methods, and results. The scientific and materials science significance of the material should be described. A short summary of synthesis or source, tests for phase purity (e.g. measured density, elemental analysis, spectra, and melting point), sample mounting, and XRD measurement and analysis methods should be included. The documentation should follow the guidelines presented in Section V (Manuscript Preparation) below. In addition to the submitted manuscript, the raw data file (as CIF) for the diffraction pattern must be provided as supplemental material. If a crystal structure or Rietveld refinement result is reported, the associated CIF must also be provided as supplemental material. If the crystal structure is already known, publication of the powder pattern needs to be justified.

(4) Rapid Communications are short articles on original research, usually limited to two printed pages of about 1000 words. They may include the same topics as in New
Diffraction Data, but are designed for rapid processing and publication. The minimum requirements for a rapid communication are: importance of the phase, chemical name, chemical formula, unit cell parameters, sample description (such as phase synthesis or source, purity, crystal habit, and supportive materials such as spectra, melting point, measured density, and elemental analysis), instrument description, and data collection parameters. In addition to the submitted manuscript, the raw data file (as CIF) for the diffraction pattern must be provided as supplemental material. If a crystal structure or Rietveld refinement result is reported, the CIF must also be provided as supplemental material.

(5) Data Reports are short articles on original research, usually limited to two printed pages of about 1000 words and are intended to quickly communicate new results for an ongoing study. Examples could be, previously unreported powder diffraction data for a material with a known crystal structure, new diffraction data for a novel material where crystal structure elucidation will be a future activity, or a previously unknown phase based on the diffraction data for a material at high temperature or pressure. The minimum requirements for a Data Report are: importance of the phase, chemical name, chemical formula, sample synthesis or source, sample mounting, instrument description, and data collection parameters. In addition to the submitted manuscript, the raw data file (as CIF) for the diffraction pattern must be provided as supplemental material.

We at the International Centre for Diffraction Data and Cambridge University Press thank the authors and editors for their contributions to the journal.

Nicole Ernst Boris Managing Editor, Powder Diffraction 